\title{
Lipid abnormalities in type 2 diabetes mellitus patients in Sri Lanka
}

\author{
Lukshmy Menik Hettihewa ${ }^{1,3}$, Sudari Wijewickrama Gunasekera ${ }^{1}$, Sudheera Sammanthi \\ Jayasinghe $^{1}$, Shalika Palangasinghe ${ }^{1}$, Thilak Priyantha Weerarathna ${ }^{2}$, Imendra Kotapola ${ }^{4}$ \\ ${ }^{1}$ Department of Pharmacology, ${ }^{2}$ Department of Medicine, ${ }^{3}$ Molecular Science and Biomedical Unit, \\ ${ }^{4}$ Department of Physiology, Faculty of Medicine, University of Ruhuna, Galle.
}

\begin{abstract}
Background \& Objective: Previous studies suggest that the prevalence of dyslipidaemia in type 2 diabetes is high with respect to the general population. But data related to the prevalence of dyslipidaemia in diabetic population is limited in Sri Lanka. This study was planned to identify the prevalence of abnormalities in lipid profile among type 2 diabetic population.
\end{abstract}

Methods: All subjects were studied for their quantitative lipid abnormalities after an overnight fast. Their anthropometric indices were measured and fasting blood sample was collected for glucose and lipids determinations.

Results: Mean value of BMI was $23.67 \pm 0.58$ $\mathrm{kg} / \mathrm{m}^{2}$ and FBS was $179.3 \pm 9.98 \mathrm{mg} / \mathrm{dL}$. There were $67 \%$ patients with high TC levels and $44 \%$ of patients with high triglyceride levels. Increased LDL levels were found in $51 \%$ of patients and $19 \%$ of patients had lower HDL levels both in males \& females. We found that $65 \%$ of patients had more than one risk factor in lipid profiles. Further, $74 \%$ of patients had combination of significantly high TC and low HDL levels.

Interpretation \& Conclusion: Contrary to the previous research findings, total cholesterol is the most common lipid abnormality in type 2 diabetics. Considering our different prevalence rates of dyslipidaemia among diabetic patients, we strongly suggest to promote investigations related to the effects of abnormalities of total cholesterol on the development of insulin resistance, the major causative factor for type 2 diabetes.

\section{Introduction}

The prevalence of type 2 diabetes has reached epidemic proportions in the US and worldwide, and is projected to increase dramatically [1].
Furthermore, the prevalence of insulin resistance, a major causative factor in the early development of type 2 diabetes and an independent risk factor for cardiovascular disease and the metabolic syndrome $\mathrm{X}$, is even more widespread [1]. The prevalence of dyslipidaemia in type 2 diabetes is double with respect to the general population [2]. These are more complex abnormalities that, in general, are caused by the interrelation among obesity, insulin resistance and hyperinsulinism [3-5]. With the treatments and tight glucose controls, blood lipid levels may improve; however, almost never do they reach normal levels. Lipid management in diabetes should begin parallel with the best possible blood glucose control, and if there is obesity, weight loss should be encouraged [5].

The quantitative and qualitative abnormalities of lipids in diabetic patients are numerous. The most common abnormality found in diabetes is high triglycerides (TG) with low high density lipoprotein (HDL); and although if low density lipoprotein (LDL) might not be higher, its metabolism is abnormal [6-8]. At present, there is enough evidence to claim that at least half of type 2 diabetics already present with some form of a macrovascular disease by the time their disease is diagnosed [4-5].

Data related to the prevalence of dyslipidaemia (qualitative lipid analysis) among diabetic patients is limited in Sri Lanka. Because of the numerous variations in quantitative and qualitative abnormalities of lipids in diabetic patients, we conducted this study to analyse the lipid profiles in patients with type 2 diabetes mellitus. Main objective was to find out the prevalence rate and the qualitative analysis of lipid abnormalities among type 2 diabetics in a Sri Lankan population. 


\section{Materials and Methods}

Selection of patients: Subjects were selected from type 2 diabetic patients that participated in the Hospital medical clinic. 43 patients were selected by convenient sampling irrespective of their treatment strategies for diabetes. Study protocol and objectives of the study were thoroughly explained to them. They were recruited after obtaining the informed written consent. Clinical history was documented and following exclusion criteria were used; age out side the range of 20-65 years, liver, kidney or cardiac failure, neoplasm and patients who are on any type of anti lipidaemic therapy.

Study protocol: The study protocol was reviewed and approved by the Ethics Committee of Faculty of Medicine, University of Ruhuna. Each individual was investigated in our laboratory after an overnight (12-14 h) fast to determine fasting blood sugar, plasma lipids and insulin levels. Their weight and height were also recorded.

Biochemical analysis: The plasma was separated immediately using centrifugation at $4000 \mathrm{rpm}$ for 10 minutes. Fasting blood glucose concentration was assessed by absorbance method (Diagnostica - Merck). Analysis of lipid was done either immediately or during first week after conservation at $-20^{\circ} \mathrm{C}$. Total cholesterol (TC) and triglyceride (TG) levels were measured enzymatically (LABKIT-P\&T Diagnostics, Spain) [3]. HDL cholesterol was measured in the supernatant after lipoprotein containing apolipoprotein B were precipitated by phostungstate/magnesium chloride solution [3]. LDL Cholesterol was calculated by Friedewald formula: LDL-cholesterol $=$ Total cholesterol (Triglycerides/5) - HDL cholesterol [3]. Serum insulin levels were determined by enzyme linked immunoabsorbent assay (ELISA) using commercial ELISA kits (DiagnosticAutomation) Body mass index (BMI) was calculated using weight and height $\left(\mathrm{kg} / \mathrm{m}^{2}\right)$.

Statistical Analysis: For the descriptive statistics after having checked the normality of the variables using the Kolmogorov-Smirnov test, the usual central and dispersion methods were used: average, SD, and 95\% CI. Power and sample size calculations were carried out based on the results of the current study, comparing changes in FI, IR, BW and BMI in 3 month of PIO allowing declaration of a difference before and after in same treatment group, at a significance level $=0.05$, with power of $80 \%$. The statistical significance of differences between the means were evaluated using the paired Student's T-test in the case of normal distribution of data sets, and using the Kolmogorov-Smirnov test when at least in one of the data sets the normal distribution was excluded. Correlation between two variables was studied with the Spearman rank-order. All statistical analyses were performed using Microcal origin 4.1(2005) and Microsoft Excel whenever applicable.

\section{Results}

Table 1 shows the baseline values of age, BMI and fasting blood sugar. Age distribution of our study sample was 26 years - 65 years (mean+/SEM, 46+/-1.5 years). Their mean body mass index was $23.7+/-0.6 \mathrm{~kg} / \mathrm{m}^{2}$ and the mean fasting blood sugar was $179.3+/-10 \mathrm{mg} / \mathrm{dL}$ (mean $+/$ SEM). Figure 1 shows $33 \%$ of diabetic patients are within 51 - 60 year of age range and $31 \%$ of them are within 41-50 year range. Figure 2 indicates most of our patients are not obese $(61 \%)$ and the BMI of study group vary from 14.8 $-33.6 \mathrm{~kg} / \mathrm{m}^{2}$.

Table 1- Anthropometric and metabolic characteristics of patients with type 2 diabetes

\begin{tabular}{ll}
\hline Basic characteristics & Mean \pm SEM $(\mathrm{n}=43)$ \\
\hline Age $($ years $)$ & $44.9 \pm 0.34$ \\
BMI $(\mathrm{kg} / \mathrm{m} 2)$ & $23.7 \pm 0.58$ \\
FBS $(\mathrm{mg} / \mathrm{dL})$ & $179.3 \pm 9.98$ \\
\hline
\end{tabular}

Qualitative and quantitative analysis of lipid profile in our study group is shown in table 2 . Total cholesterol, triglycerides, HDL cholesterol and LDL cholesterol were $246.5+/-7.6,156.3+/-$ $6.2,57.2+/-1.6$, and $157.2+/-7.5 \mathrm{mg} / \mathrm{dL}$ respectively (Mean+/-SEM). Total cholesterol to HDL ratio (TC/HDL ratio) was $4.43+/-0.18$ (Mean+/-SEM). Prevalence of dyslipidaemia was diagnosed by cut points, mentioned in the diagnostic kit [3] (LABKIT- P\&T from Spain). 
Hypercholesterolemia was considered when the total cholesterol was ?220 mg/dL and TG \& LDL cholesterol levels were considered to be abnormal if they were $\geq 150 \mathrm{mg} / \mathrm{dL}$ [3]. HDL cholesterol concentration was considered to be abnormal if it was ? $40 \mathrm{mg} / \mathrm{dL}$ in males and $\leq 50$ $\mathrm{mg} / \mathrm{dL}$ in females [3]. Total cholesterol/HDL ratio was considered to be as a risk when it is $\geq 3.8$ in males and $\geq 3.1$ in females.

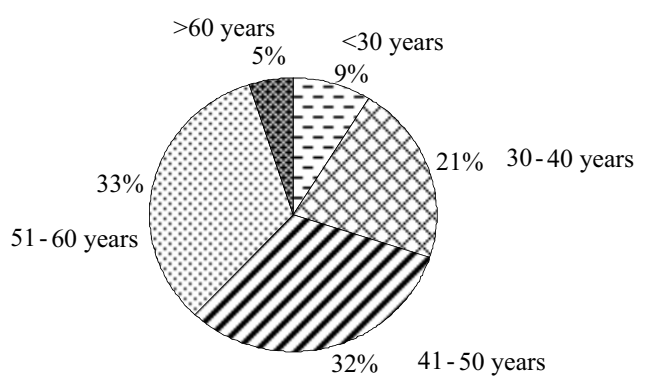

Figure 1 - Age distribution among patients with type 2 diabetes mellitus

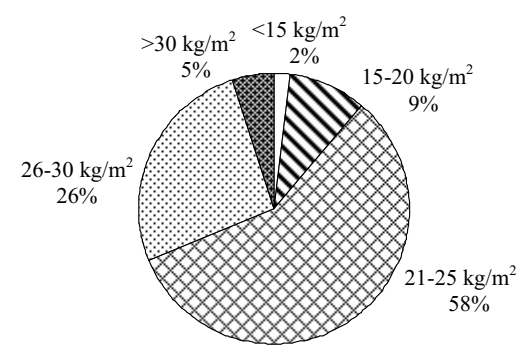

Figure 2 - BMI distribution among patients with type 2 diabetes mellitus

Next, we analysed the prevalence rate of hypercholesterolaemia, hypertriglyceridaemia, low HDL and high low density lipoproteinaemia among our study group. Figure 3 shows that $74 \%$ of patients had high TC / HDL ratio and $67 \%$ of patients had isolated hypercholesterolaemia in our study group. In addition, $44 \%$ of them had hypertriglyceridaemia $19 \%$ of patients found to be with abnormally low HDL levels and 51\% patients with abnormal LDL levels (Figure 3). In our type 2 diabetic group, the most common abnormality was the high TC / HDL cholesterol ratio. 22 out of 24 females had TC/HDL ratio $>$ 3.1 and 10 males had TC / HDL ratio $>3.8$ patients (91\% for females, $53 \%$ for men and $74 \%$ for both genders). We have found that second most common abnormality was isolated hypercholesterolaemia ( $63 \%$ of females, $74 \%$ of males and $67 \%$ of both genders). Our results further indicate that abnormal LDL cholesterol levels were detected in $47 \%$ of men, $54 \%$ of women and $51 \%$ of both genders. Similarly, hypertriglyceridaemia was found in $37 \%$ of men, $50 \%$ of women and $44 \%$ of both genders. The prevalence of having more than one risk factor in lipid profiles was $65 \%$ in our study group (data not shown).

Table 2 - Quantitative and qualitative analysis of lipid profile before the commencement of the treatment. Values expressed as mean and standard error of mean (SEM)

\begin{tabular}{ll}
\hline Parameters of lipid profile & Mean \pm SEM \\
\hline Total cholesterol $(\mathrm{mg} / \mathrm{dL})$ & $246.5 \pm 7.6$ \\
Triglycerides $(\mathrm{mg} / \mathrm{dL})$ & $156.3 \pm 6.2$ \\
HDL cholesterol $(\mathrm{mg} / \mathrm{dL})$ female & $57.0 \pm 2.1$ \\
HDL cholesterol $(\mathrm{mg} / \mathrm{dL})$ male & $57.5 \pm 2.5$ \\
LDL cholesterol $(\mathrm{mg} / \mathrm{dL})$ & $157.2 \pm 7.5$ \\
TC/HDL ratio female & $4.3 \pm 0.2$ \\
TC/HDL ratio male & $4.6 \pm 0.3$ \\
\hline
\end{tabular}

\section{Discussion}

Type of dyslipidaemia reported among diabetic population is numerous in different places in world indicating that dyslipidaemia can be influenced by the interaction of genetic and environmental factors [8]. We are in agreement with the previous reports that dyslipidaemia is a common association with type 2 diabetic patients $[2,4]$. Though most of diabetic patients are not obese ( $61 \%), 75 \%$ of them had elevated TC/HDL ratio and $67 \%$ of them had isolated hypercholesterolaemia. Addition, 51\% of patients had high LDL cholesterol levels (figure 2 ). According to our results, prevalence of TC/HDL cholesterol ratio was higher in female diabetic patients [9]. The prevalence of the lipid abnormalities reported by Mexican nationwide survey done by Carlos A and Aguilar S. et al [8] is similar to that observed in Turkish [10] and other Asian populations, including Bangladeshi and Pakistani populations [11] had a different view from our results. Their study shows that $54 \%$ of the diabetic population had isolated hypertriglyceridaemia [8] and $26 \%$ patients with type 2 diabetes mellitus had combination of low HDL cholesterol $(<0.9 \mathrm{mmol} / \mathrm{l}, 35 \mathrm{mg} / \mathrm{dl})$ with hypertriglyceridaemia $(\geq 2.26 \mathrm{mmol} / \mathrm{l})$. We are in agreement with the above report in relation to prevalence rates of isolated hyper- 
triglyceridaemia among diabetic population (44\% in ours and 54\% in Mexican nationwide survey) [8]. They have found out isolated hypertriglyceridaemia $(\geq 2.26 \mathrm{mmol} / \mathrm{l})$ is the most common lipid abnormality among diabetic population but in contrary, our results show the most common lipid abnormality is the high TC/HDL ratio (74\%). We also found that $67 \%$ of patients showing isolated hypercholesterolaemia and $51 \%$ of patients having high LDL levels. This observation further confirms that patients with diabetes had co-incidence of several abnormal lipid profiles.

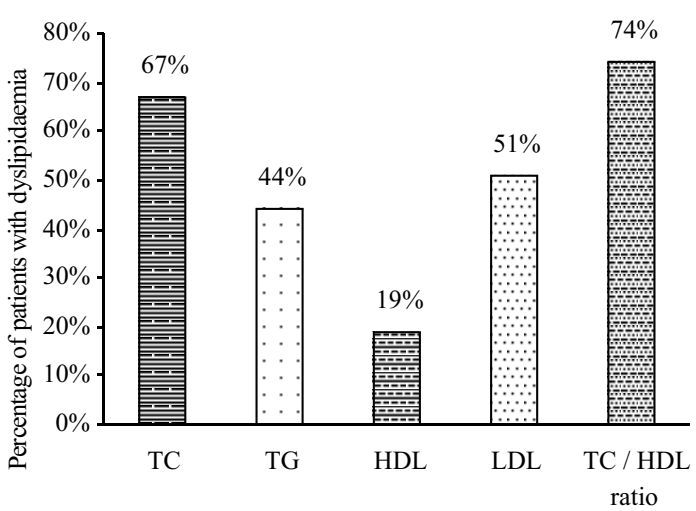

Figure 3 - Percentage of patients with abnormal lipid profiles

In conclusion, our data show that the most common abnormal lipid profile among type 2 diabetic patients is hypercholesterolaemia, confirmed by high prevalence rate of total cholesterol/HDL ratio $(74 \%)$ and isolated hypercholesterolaemia (67\%). We have recently found out that there is a significant genetic association between development of insulin resistance and total cholesterol levels among type 2 diabetic patients [12]. It further confirms that total cholesterol could have impact on the development of insulin resistance in type 2 diabetes mellitus. Considering all the finding in our previous and current studies, we strongly think in investigating the possible metabolic association of between insulin resistance and abnormal levels in cholesterol in type 2 diabetic patients.

\section{Acknowledgements}

We gratefully acknowledge Mrs. N. Samaranayaka and Mrs. A.G. Punyalatha in the
Department of Pharmacology, Faculty of Medicine University of Ruhuna for their assistance with laboratory analysis.

\section{References}

1. Evans JL, Rushakoff RJ. Oral Pharmacological Agents for Type 2 Diabetes: Sulfonylureas, Meglitinides, Metformin, Thiazolidinediones, $\alpha$-Glucosidase Inhibitors, and Emerging Approaches. In: Endotext.com; Diabetes and Carbohydrate Metabolism. Degroot L, Goldfine ID, Rushakoff RJ, Eds. Available from; http://www.mdtext.com/ diabetes/diabetes 16/diabetesframe16.htm, MDtext.com, 2002.

2. Haffner SM. Management of dyslipidaemia in adults with diabetes. Diabetes Care 1998;21: 160-78.

3. Burstein M, Selvenick HR, Morfin RJ. Journal of Lipid Research 1970; 11: 583, and (1970) Koeler DF, Azar MM and Cols. Clin Chem 22, 98, (1976) Lopez-Virella M. Clin Chem 23, 882, (1977) Frienewald WT. Clin Chem 14, 499 (1972).

4. American Diabetes Association - Position Statement. Management of Dyslipidemia in Adults with Diabetes. Diabetes Care 1998; 21: 179-82.

5. American Diabetes Association - Position Statement. Management of Dyslipidemia in Adults with Diabetes. Diabetes Care 2001;24 Supp1.

6. Purnell JQ, Brunzell JD. Effect of intensive diabetes therapy on diabetic dyslipedaemia. Diabetes Reviews 1997; 5: 434-44.

7. Steiner G. Lipid intervention trials in diabetes. Diabetes Care 2000; 23: 49-53.

8. Carlos A, Aguilar S, Gustavo O, et al. High prevalence of low HDL cholesterol concentrations and mixed hyperlipidemia in a Mexican nationwide survey. Journal of Lipid Research 2001; 42: 1298-307.

9. British National Formulary. $41^{\text {st }}$ ed. Joint British Societies Coronary risk prediction chart (reprinted and modified with permission from Heart 1998; 80: 1-29); March 2001.

10. Mahley RW, Erhan-Palaoglu K, Atak Z, et al. Turkish heart study: lipids, lipoproteins and apolipoproteins. Journal of Lipid Research 1995; 36: 839-59.

11. Bhopal R, Unwin N, White M, et al. Heterogeneity of coronary heart disease risk factors in Indian, Pakistani, Bangladeshi, and European origin populations: cross sectional study. British Medical Journal 1999; 319:215-20.

12. Menik HL, Sammanthi JS, Priyantha WT, et al. Significant genetic association between insulin resistance and total cholesterol in type 2 diabetes mellitus; Preliminary study. OJHAS. http://www.ojhas.org/issue13/2005-1-4.htm 